\title{
ORIGINAL ARTICLE Hydrodynamic immunization leads to poor CD8 T-cell expansion, low frequency of memory CTLs and ineffective antiviral protection
}

\author{
N Obeng-Adjei, DK Choo and DB Weiner
}

\begin{abstract}
Hepatotropic pathogens, such as hepatitis B (HBV) and hepatitis C (HCV), often escape cellular immune clearance resulting in chronic infection. As HBV and HCV infections are the most common causes of hepatocellular carcinoma (HCC), prevention of these infections is believed to be key to the prevention of HCC. It is believed that an effective immune therapy must induce strong cytotonic T lymphocytes (CTLs) that can migrate into the liver, where they can clear infected hepatocytes. Here, we compared the induction of CD8 T cells by two different DNA immunization methods for T-cell differentiation, function, memory programming and their distribution within relevant tissues in a highly controlled fashion. We used hydrodynamic tail vein injection of plasmid to establish liver-specific LCMV-gp antigen (Ag) transient expression, and studied CD8 T cells induced using the P14 transgenic mouse model. CD8 T cells from this group exhibited unique and limited expansion, memory differentiation, polyfunctionality and cytotoxicity compared with T cells generated in intramuscularly immunized mice. This difference in liver-generated expansion resulted in lower memory CD8 T-cell frequency, leading to reduced protection against lethal viral challenge. These data show an unusual induction of naive CD8 T cells contributed to the lower frequency of Ag-specific CTLs observed after immunization in the liver, suggesting that limited priming in liver compared with peripheral tissues is responsible for this outcome.
\end{abstract}

Cancer Gene Therapy (2013) 20, 552-563; doi:10.1038/cgt.2013.52; published online 23 August 2013

Keywords: DNA vaccine; intrahepatic; intramuscular; immunization; CD8 T cells

\section{INTRODUCTION}

A key feature of adaptive immunity underpinning the basis of immunization is the ability of the immune system to mount a specific response against a particular pathogen's antigens (Ags), leading to induction of long-lived memory cells that are capable of immune clearance and protection upon reinfection. ${ }^{1,2}$ In general, naive adaptive immune cells are activated when they meet a professional Ag-presenting cell bearing their specific $\mathrm{Ag}$ in secondary lymphoid organs. ${ }^{3,4}$ Secondary lymphoid organs have highly organized structures allowing for the concentration of lymphocytes, which in turn enhances the access of Ag-presenting cells to specialized $B$ and $T$ cells for the initiation of adaptive immunity, involving activation, proliferation and expansion, ${ }^{5-7}$ as well as the subsequent development of protective effector cells. The activation of lymphoid-resident naive T cells by Ag-presenting cell grants the T-cell the ability to deploy to non-lymphoid tissues that are connected to the lymphoid where the lymphocyte encountered the Ag. A number of independent groups have suggested that the microenvironment of the lymphoid tissue serving as the activation site present homing instructions to the newly activated lymphocyte. ${ }^{8-10}$ This theory was further supported by the observation that Peyer's patch dendritic cells imprint gut-homing specificity on $T$ cells and drive $T$ cells to preferentially migrate into the small intestine. ${ }^{11}$

Despite this knowledge of selective homing of lymphocytes, few studies have been performed to show the link between $\mathrm{Ag}$ placement and effector lymphocyte migration preference. ${ }^{12}$ With more data supporting the importance of cytotonic T lymphocytes
(CTLs) during viral clearance in hepatotropic infections, such as hepatitis B and hepatitis $C$, it has become clear that preferential migration of $\mathrm{T}$ cells into the liver in these infections may be important. These two important infections are major causes and risk factors for HCC or liver cancer, the third most common and the most deadly cancer. HCC claims the lives of such individuals within 5 years of diagnosis, and is a major health issue in countries where HBV and HCV are endemic. Efforts directed toward the development of therapeutic vaccines against these pathogens have yet to yield fruitful results. Therefore, it is important to take a step back and evaluate other factors that may influence the induction of strong immune responses against liver-tropic pathogens, which may prove critical for the development of effective immune therapies.

In this study, we qualitatively and quantitatively compared CD8 $T$ cells activation when Ag encoded by a DNA vaccine is delivered either intrahepatically (I.H.) or intramuscularly (I.M.). We used hydrodynamic injection (HI) to transiently transfect the LIV of naive P14.Thy1.1-recipient mice with an lymphocytic choriomeningitis virus glycoprotein (LCMV-gp) transgene and followed CD8 $T$ cells as they developed from their initial priming through their effector and memory phases. We examined their initial clonal expansion, functional development, development of cytotoxicity and development into long-lived memory cells, and, most importantly, their frequency within lymphoid and non-lymphoid tissues.

We observed no difference in the initial priming, functionality or long-term memory T-cell distributions between immunization strategies at either site. However, significant differences based on 
immunization location were observed in clonal expansion, effector/memory differentiation and induction of CTLs. Further investigations revealed that CD8 T cells from the hydrodynamic LIV-immunized group were being primed outside the draining lymph nodes. This priming choice induced defective CD8 T-cell expansion and the unique development of short-lived (SLEC) and memory precursor effector cells (MPECs). Although further studies are needed, these data highlight the importance for vaccine approaches that will preferentially traffic effective immune cells into the LIV without the need to place Ags in the LIV for the development of effective immunity.

\section{MATERIALS AND METHODS}

Mice

C57BL/6 mice (CD90.2) were purchased from Jackson Laboratories (Bar Harbor, ME, USA). CD90.1 + P14 mice bearing the DbGP33-specific T-cell receptor were a gift from Dr John Wherry of University of Pennsylvania. All mice were maintained in specific pathogen-free facilities at the University of Pennsylvania. The University of Pennsylvania Institutional Animal Care and Use Committee approved all protocols, and all experiments were performed according to the guidelines of the University of Pennsylvania Institutional Animal Care and Use Committee.

\section{P14 chimeric}

Mice were generated by adoptively transferring $1 \times 10^{5}$ naive T-cell receptor transgenic T cells into naive $\mathrm{B} 6$ mice (referred to as P14 chimeras). For the FTY720 experiment, FTY720 was dissolved in sterile water at $2.5 \mu \mathrm{g} \mathrm{ml}^{-1}$ for treated mice, whereas control mice received normal drinking water. ${ }^{13}$ For L-selectin experiments, treated mice intravenously (i.v.) received $200 \mathrm{~g} \mathrm{ml}^{-1}$ of the MEL-14 (anti-CD62L) blocking antibody i.v. ${ }^{14} 4 \mathrm{~h}$ before and $24 \mathrm{~h}$ after the adoptive transfer of P14 cells.

\section{DNA plasmid vector}

The plasmid construct was a gift from Dr Rafi Ahmed of Emory University, Atlanta, GA, USA. It contains the full-length LCMV-GP with five mutations. Only two of the mutations are in a specific epitope sequence. One of those two mutation changes the ending of the GP33 epitode ending by replacing methionine with cysteine.

GP33: KAVYNFATM $\rightarrow$ KAVYNFATC

GP92: CSANNSHHY $\rightarrow$ SANNPHHY

\section{Immunization}

For hydrodynamic tail vein immunization (HDTV), mice were injected i.v. with $200 \mu \mathrm{g}$ of plasmid in $2 \mathrm{ml}$ (about $10 \%$ volume of the mouse weight) of PBS solution within $7 \mathrm{~s}$ to transiently transfect the LIV. ${ }^{15}$ Intramuscular (I.M.) immunization was done by either directly injecting $200 \mu \mathrm{g}$ or electroporating $20 \mu \mathrm{g}^{16}$ of plasmid in $30 \mu \mathrm{l}$ water into the tibialis anterior of P14 chimeras.

\section{Histology}

For histology, LIVs were perfused with PBS and fixed for $24 \mathrm{~h}$ in $4 \%$ paraformaldehyde. These were then placed in $30 \%$ sucrose for $24 \mathrm{~h}$, frozen in OCT (frozen tissue matrix) and cut on a cryostat. The sectioned tissues of LIV were stained and images were obtained using a Zeiss Axiovert 100 inverted confocal microscope (Carl Zeiss, Thornwood, NY, USA). Analysis and quantification of florescence intensities were conducted using Image $J$ software (National Institute of Health, Rockville, MD, USA).

\section{Antibodies, flow cytometry and intracellular cytokine staining}

All antibodies were purchased from BD Biosciences (San Jose, CA, USA) except antibodies to mouse CD127, KLRG1 and PD-1, which were purchased from eBiosciences (San Diego, CA, USA). Major histocompatibility complex class I peptide tetramer to LCMV-gp33 were a gift from Dr Rafi Ahmed's lab. Cells were stained for surface or intracellular proteins as described previously. ${ }^{17,18}$ Briefly, for intracellular cytokine staining, lymphocytes $\left(10^{6}\right.$ per well) were stimulated with the indicated peptides $\left(0.2 \mu \mathrm{g} \mathrm{ml}^{-1}\right)$ in the presence of Brefeldin A and CD107a for $5 \mathrm{~h}$ followed by surface staining for CD8 and intracellular staining for interferon (IFN)- $\gamma$, tumor necrosis factor (TNF)- $\alpha$ or interleukin (IL)-2.

\section{In vivo cytotoxicity assay}

An in vivo cytotoxicity assay was performed as previously described. ${ }^{19,20}$ Briefly, splenocytes from naive mice were stained with either $1 \mu \mathrm{m}$ or $1 \mathrm{~nm}$ CFDA SE (Invitrogen, Grand Island, NY, USA). The labeled splenocytes were then coated with the indicated peptides $(1 \mu \mathrm{M})$ and $10^{7}$ cells of each population were i.v. injected into naive or immunized mice. After $24 \mathrm{~h}$, cells from the spleen and LIV were isolated and analyzed by flow cytometry. The percent killing was calculated as follows: $100-(((\%$ relevant peptide pulsed in infected)/(\% irrelevant peptide pulsed in infected))/ $((\%$ peptide pulsed in uninfected $) /(\%$ irrelevant peptide pulsed in uninfected)) $\times 100$ )

\section{Viral challenge}

For lethal challenge studies, mice were challenged intracranial (I.C.) with 200 plague-forming units of LCMV Armstrong, as previously described, ${ }^{21}$ in $30 \mu \mathrm{l}$ of RPMI. Mice were observed daily for 3 weeks, a time point known to be adequate in the LCMV I.C. challenge model. All LCMV-infected animals were housed in biosafety level-2 facilities.

\section{Statistical analysis}

Data were evaluated using the two-tailed Student's $t$-test. Results were expressed as means $+I-$ s.e. $P$ values $<0.05$ were regarded as significant.

\section{RESULTS}

$\mathrm{HI}$ of LCMV-gp plasmid established acute LIV infection

$\mathrm{HI}$ of Ag-encoded DNA has been used previously as a model for acute HBV and HCV infection. ${ }^{22}$ Depending on the type of DNA vector or promoter, ${ }^{23}$ expression of the transgene can be transient or can persist for up to several weeks. We used this model for expression of an LCMV-gp in mouse LIV (Figure 1a) after hydrodynamically injecting a CMV promoter-based plasmid DNA vector. Expression of this transgene product was exclusive to the LIV and absent in other major tissues (brain, heart, intestine, kidney, lymph nodes, lung (LUN), muscle, spleen (SPL) and skin). The expression of LCMV-gp was detectable at $16 \mathrm{~h}$ post injection and was largely lost by day 5 (Figure $1 \mathrm{~b}$ ).

Intrahepatic immunization induces equivalent activation markers and effector activities

Activation of CD8 T cells from both I.H. and I.M. immunized animals was determined by their levels of proliferation and effector phenotypic marker expressions. Five days following immunization, Ag-specific CD8 T cells were detectable as observed using $D^{\mathrm{b}} \mathrm{GP} 33$ tetramer to stain the PMBCs of these mice, indicating successful priming of naive CD8 T cells in both immunization groups. CFSElabeled adoptively transferred T cells from the I.H. model exhibited quicker homeostatic proliferation during the first week of immunization in peripheral lymph nodes (PLN) and SPL as compared with the I.M. model (Figure 2a). Interestingly, proliferation in nonlymphoid tissues such as LIV and LUN at day 7 was, however, comparable between the two groups. Ag-specific CD8 T-cell proliferation in all tissues averaged around 98\%, 2 weeks post immunization.

Next, we examined the expression levels of different activation markers, such as CD25 and CD44, and CD62L, following CD8 T-cell priming in both models. We observed a significant increase in CD25 and CD44 expression on Thy1.1 CD8 T cells from both I.H. and I.M. mice in all tissues at day 7 and 14 post infection (Figure 2b). In addition, downregulation of CD62L, which is usually seen in effector CD8 T cells, was visible 14 days post infection. The comparable proliferation and effector phenotype induced indicate similar Ag stimulation of CD8 T cells in both groups.

The next important study was to determine if I.H. CD8 T cells could induce antiviral activity in response to antigenic re-encounter. We assessed the induced T cells' ability to the produced antiviral cytokines, IFN- $\gamma$, TNF- $\alpha$ and IL-2, in response to ex vivo LCMV-GP33 peptide stimulation (Figure 2c). About 50, 55 and 13\% of CD8 $\mathrm{T}$ cells from the lymph nodes of the I.H. group produced IFN- $\gamma$, 


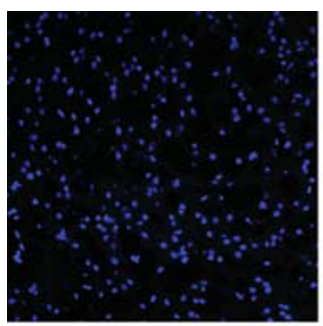

Lymph Node

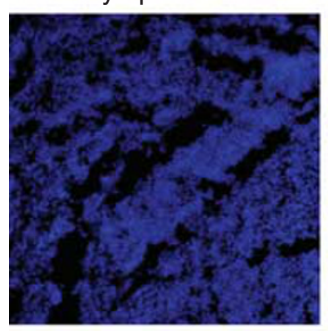

Heart

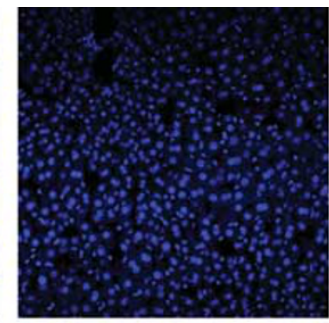

Lung

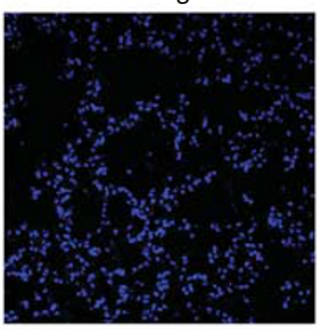

8

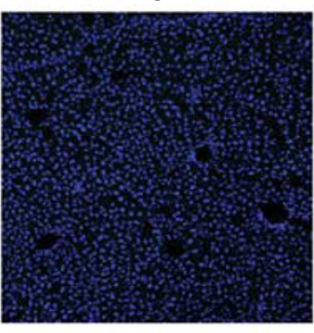

48

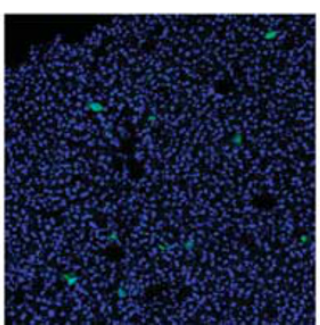

Intestine

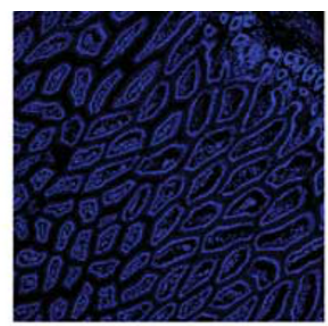

Muscle

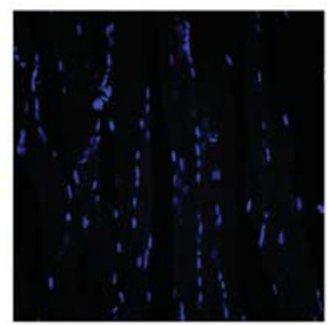

16

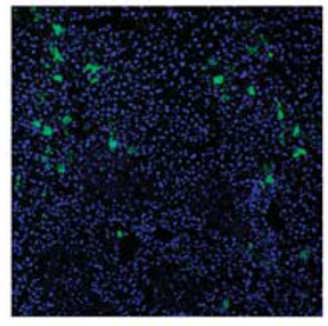

72

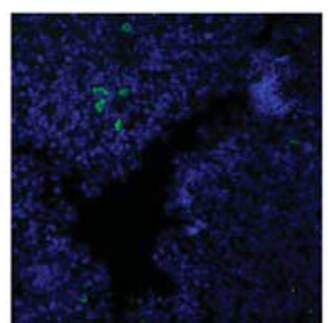

Kidney

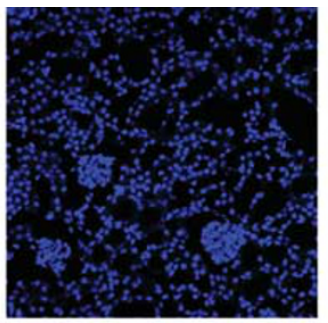

Spleen

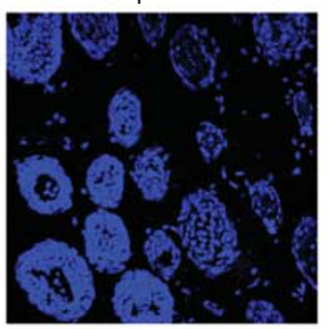

24

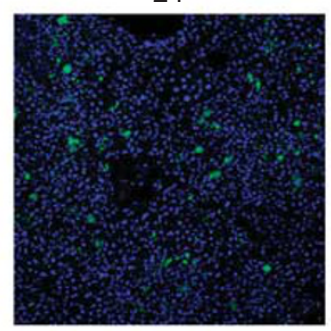

120

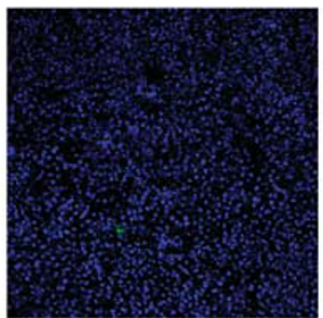

Figure 1. Transiently expressing transgene product in the liver (LIV). Mice hydrodynamically injected with LCMV-gp mutant DNA expressed Ag exclusively in the LIV. (a) Immunohistochemistry staining of the transgene product in tissue sections $24 \mathrm{~h}$ post injection with LCMV-gp mutant plasmid. Results represent brain, heart, intestine, kidney, LIV, lymph node, lung (LUN), muscle, spleen (SPL) and skin. (b) Time course of transgene transduction efficiency in mouse LIV.

TNF- $\alpha$ or IL-2, respectively, in this assay. These numbers were higher for SPL, where 77, 67 and $30 \%$ of CD8 T cells produced the above-mentioned cytokines. The LIV showed lower cytokine production at 44,59 and $7.8 \%$, respectively. In contrast, a higher percent of lymphoid-primed CD8 T cells produced these cytokines. For example, 93, 93 and 45\% of CD8 T cells from the SPL of the I.M. control group produced IFN- $\gamma$, TNF- $\alpha$ or IL-2, respectively. Nonetheless, similarities in polyfunctionality (the ability of single cells to produce multiple cytokines) were observed in the SPL and LIV of the two groups. Although, cells from the lymph nodes of the I.H. group were significantly less functional than those of the I.M. group (Figure $2 \mathrm{~d}$ ).

In addition to antiviral cytokine production, a crucial function of effector CD8 T cells is induction of cytotoxicity. We stained the Agspecific CD8 T cells for degranulation markers, granzyme B and CD107a, in order to verify whether these cells were functional effector CTLs. Both groups induced degranulation markers following Ag stimulation (Figure 2e). Accordingly, we used an in vivo cytotoxicity assay to assess the whether I.H.-immunized mice's CD8 T cells became effective CTLs. One week after immunization, we examined the killing of transferred target cells pulsed with either LCMV-GP33 peptide or LCMV-NP (control). Twenty-four hours after transfer of target cells, about $88 \%$ of LCMVGP33-pulsed target cells were eliminated in the blood of the I.H. group, but only $76 \%$ of the target cells were killed in the I.M. group (Figure 2f).

Intrahepatic immunization promptly establishes memory precursors effector CD8 T cells and maintains their memory phenotype

The ultimate goal of immunization is to generate strong effector cells to clear infection along with long-lasting memory CD8 T cells, which will quickly respond to subsequent infections. Recent studies have provided a phenotypic profile for a subset of effector 


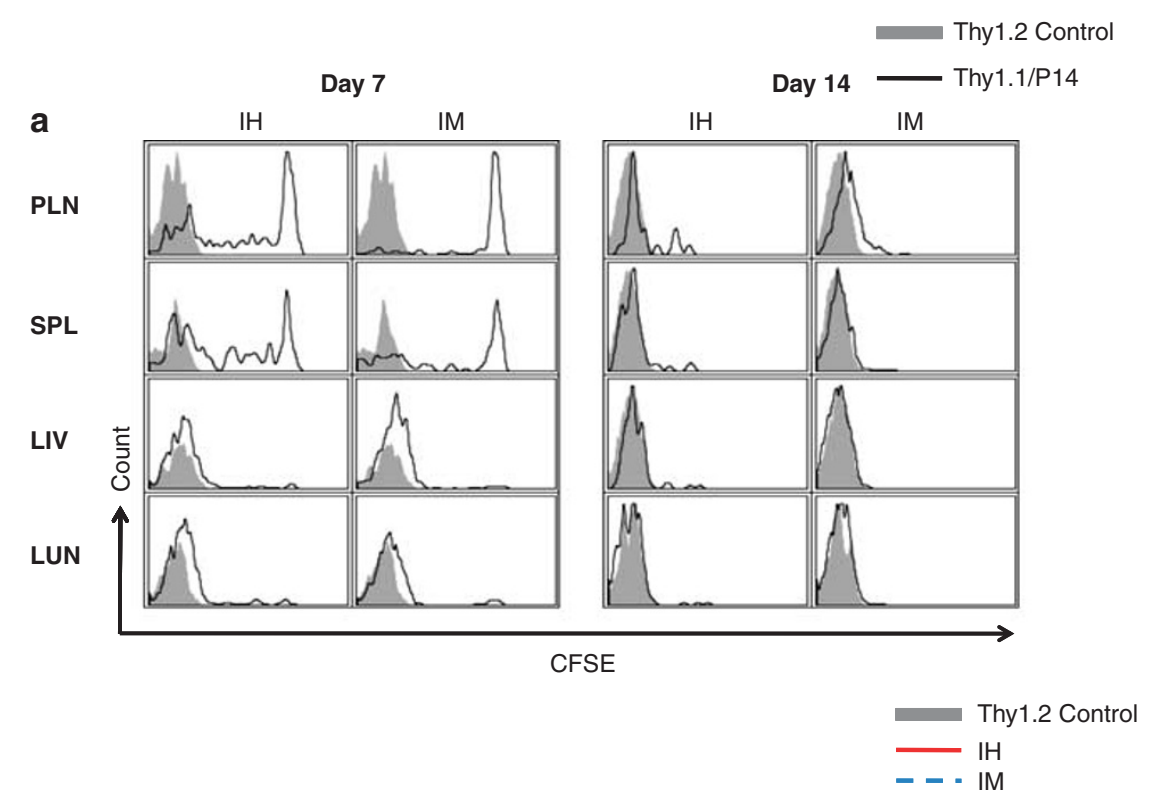

b
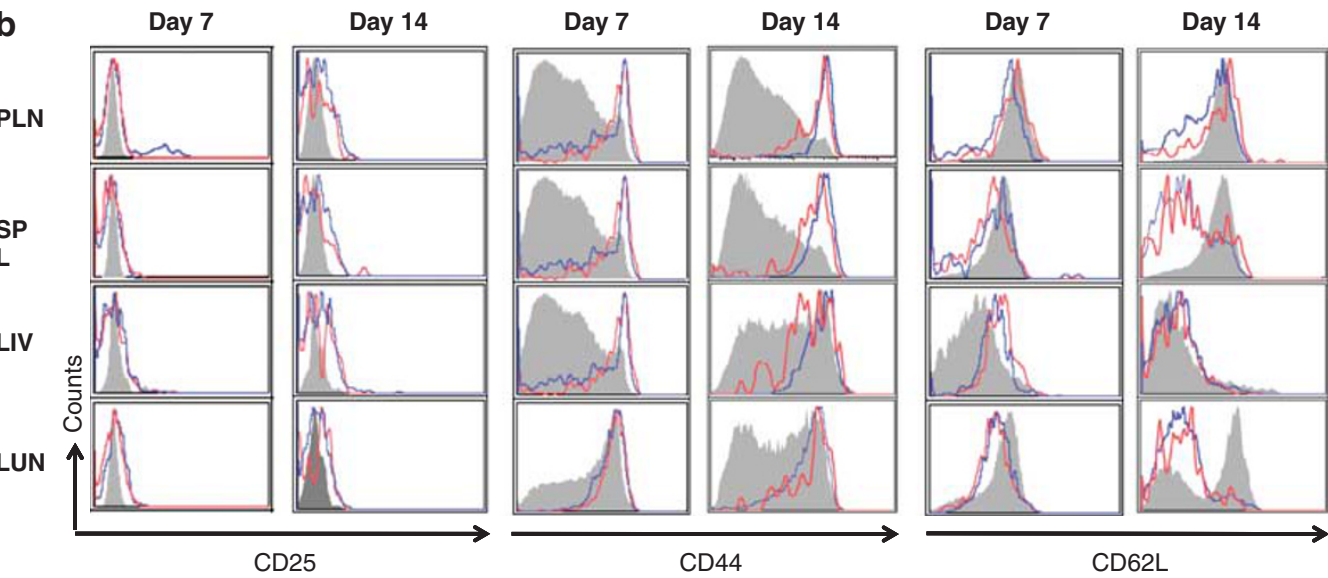

c
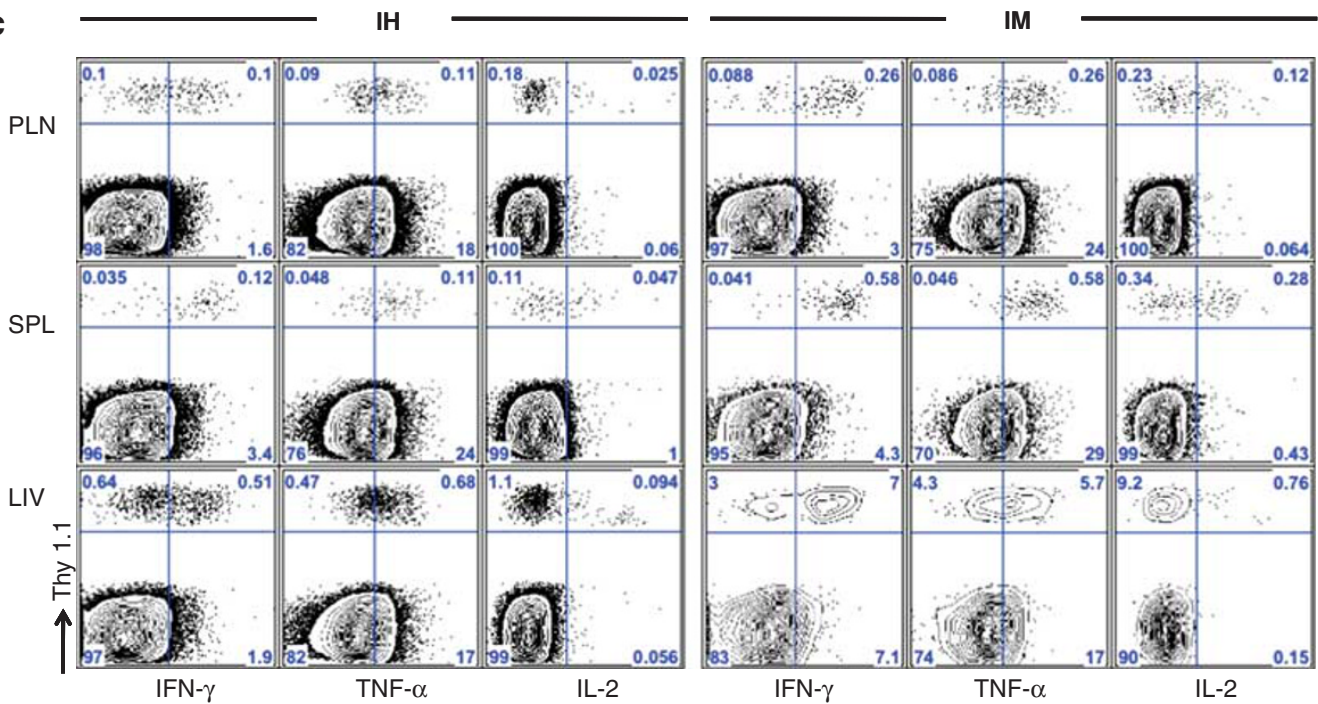

Figure 2. Effector DbGP33 ${ }^{+}$-specific CD8 T cells profile following intrahepatic (I.H.) immunization of P14 chemaric mice with LCMV-gp mutant. CFSE-labeled CD8 T cells isolated from lymphoid and non-lymphoid organs of mice either expressing Ag either I.H. or I.M. were analyzed at indicated time points. (a) Proliferative capacity and (b) expression of important activation markers on transferred $\mathrm{D}^{\mathrm{b}} \mathrm{GP} 33^{+}$-specific CD8 T cells. (c) The ability of I.H.-immunized CD8 T cells to produce cytokines: IFN- $\gamma$, TNF- $\alpha$ and IL-2, following ex vivo stimulation with synthetic peptide. (d) A representative flow chart showing IFN- $\gamma$-positive cells and their ability to coproduce TNF- $\alpha$ and IL-2 (pie chart). (e) Splenocytes from both groups (I.H. and I.M.) expressed degranulation markers (CD107a and GzmB). (f) GP33-specific CD8 T cells from both groups display rapid cytolytic activity during the first week of immunization. Data are representative of three independent experiments with four mice per group. 
d
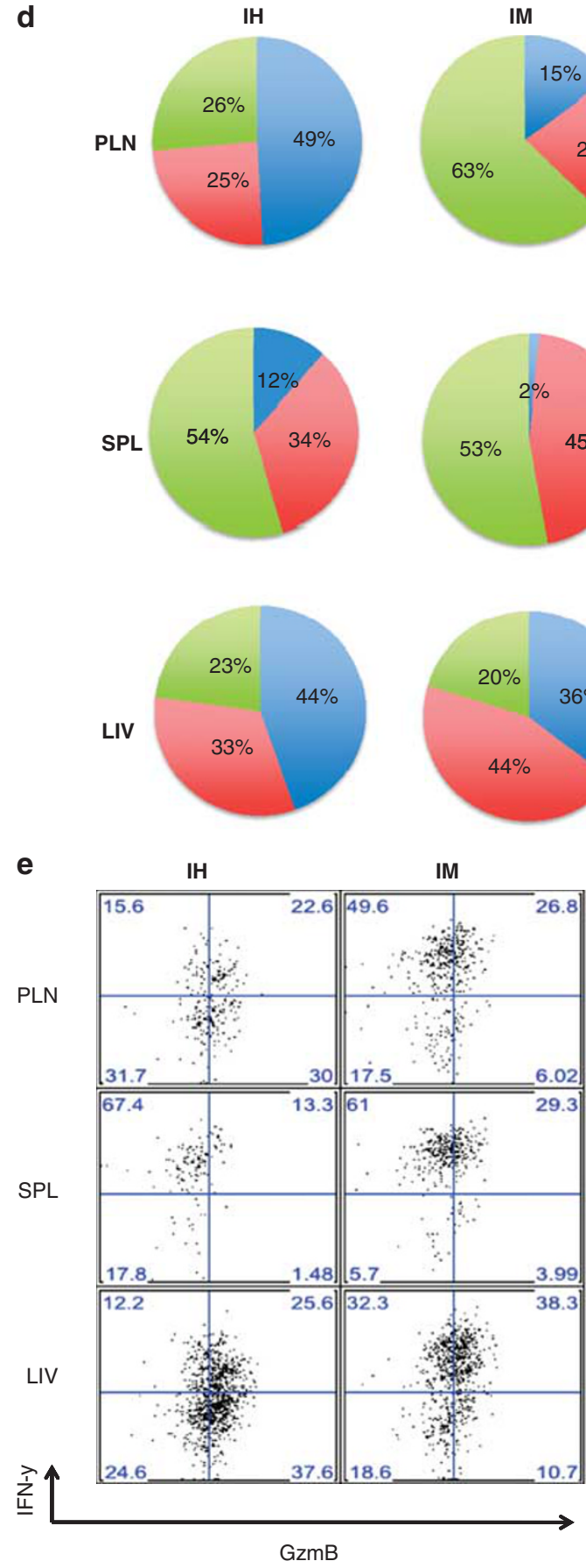

f

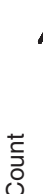

言

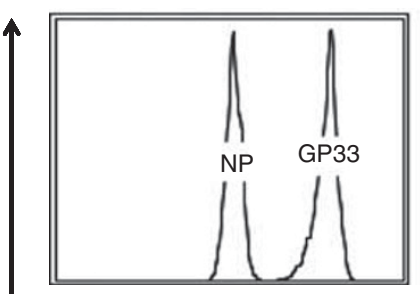

IM

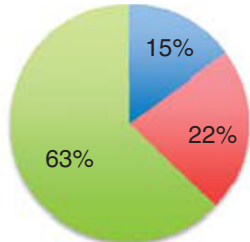

$\square$ Single Producers (IFN-y only)

Double Producers (IFN-y and TNF-a)

$\square$ Triple Producers (IFN-y, TNF-a and IL-2)
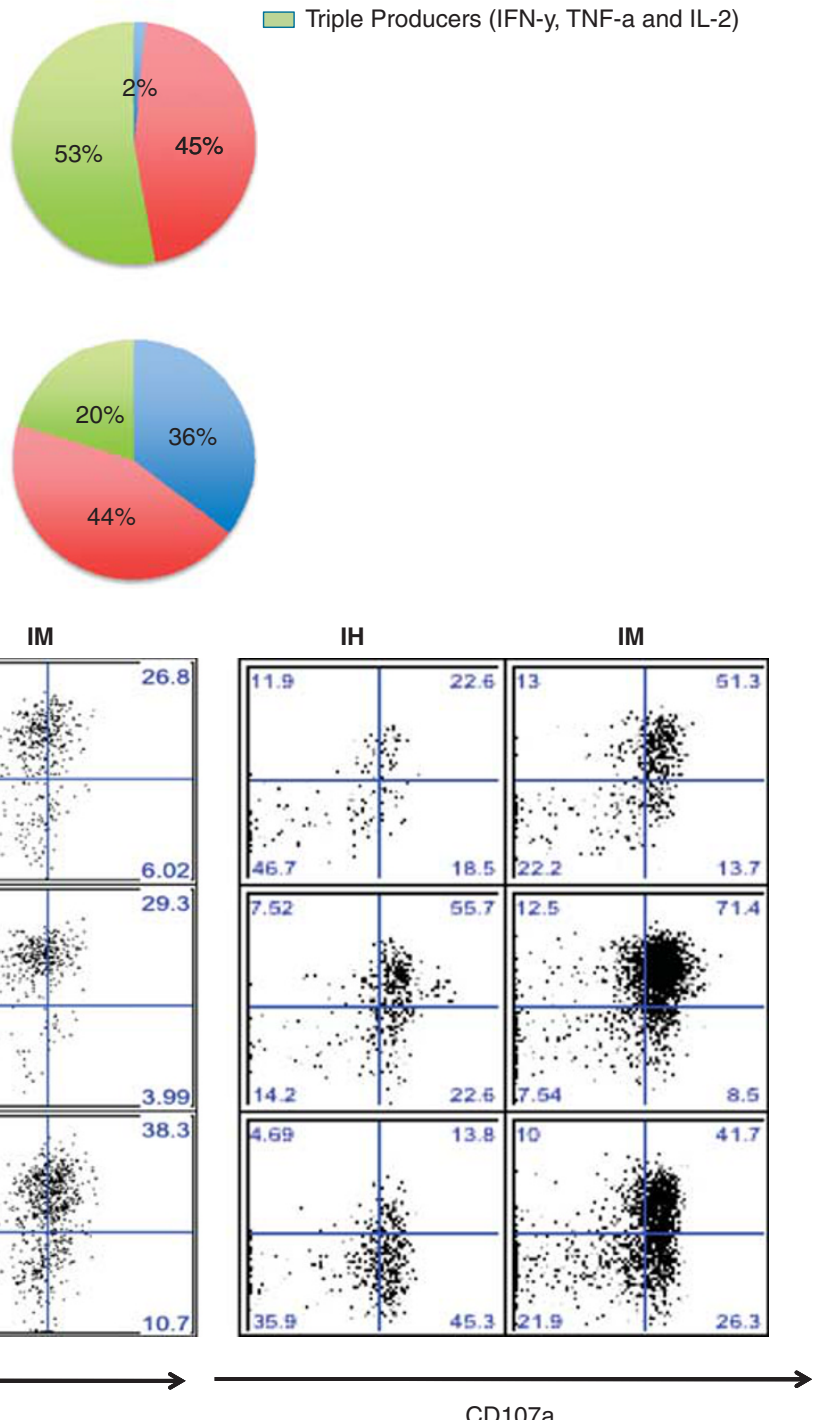

IH

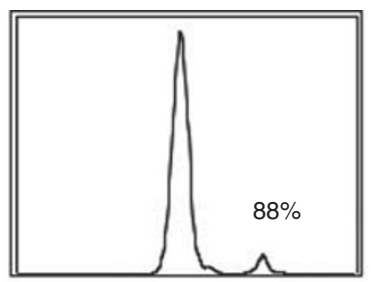

IM

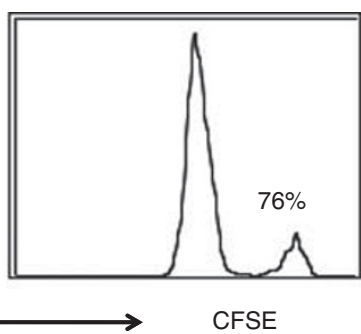

Figure 2. (Continued). 


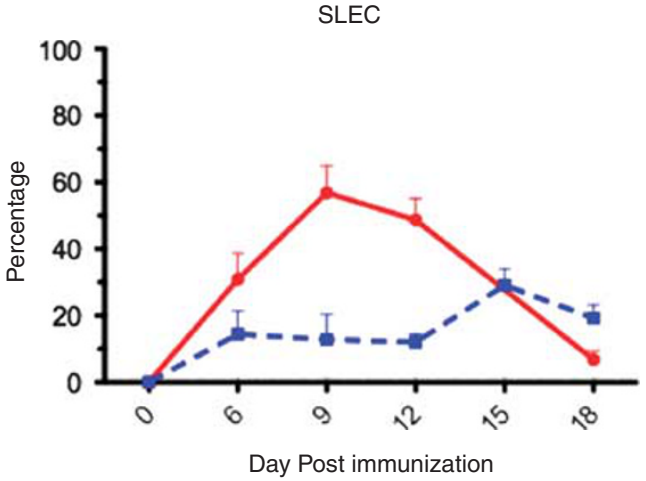

$\mathrm{IH}$

MPEC $\quad--=$ IM

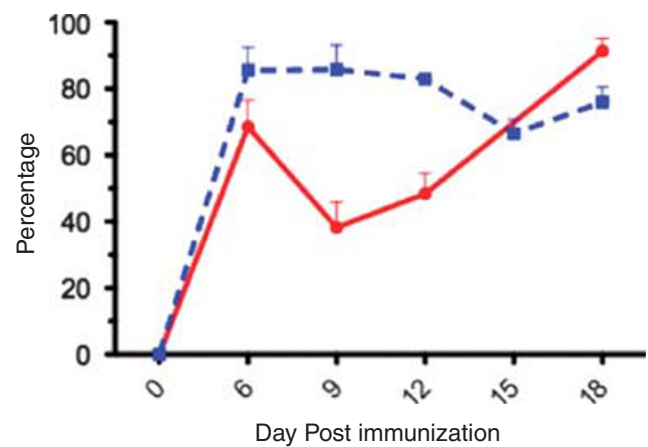

Day 7

Day 14

Day 21
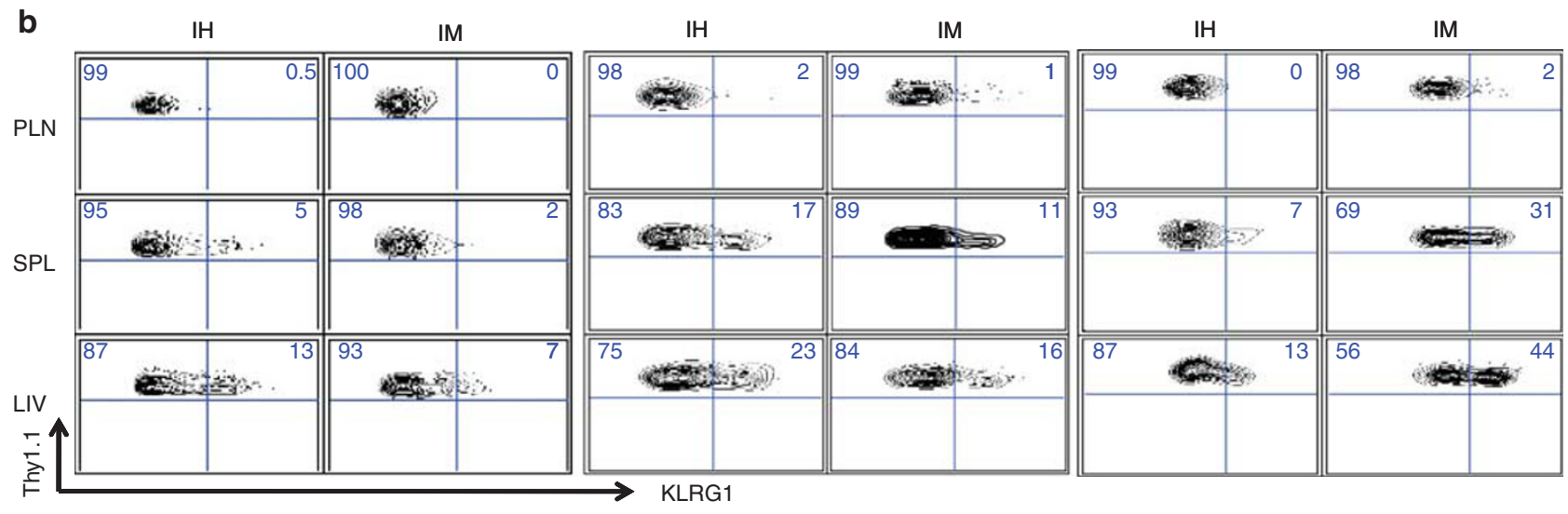

C

PLN

SPL
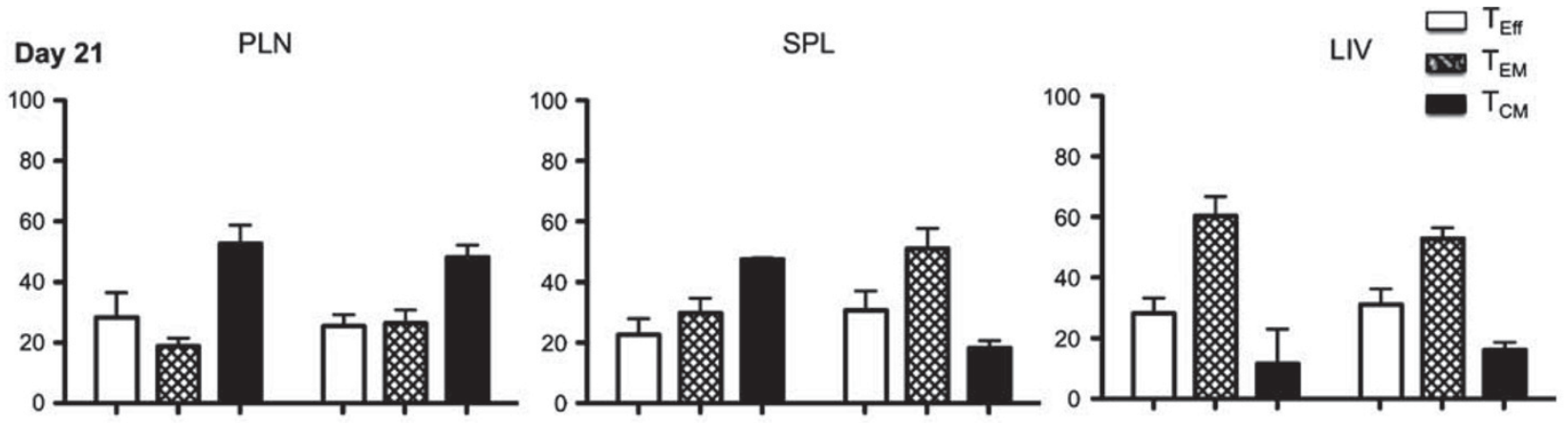

Day 84
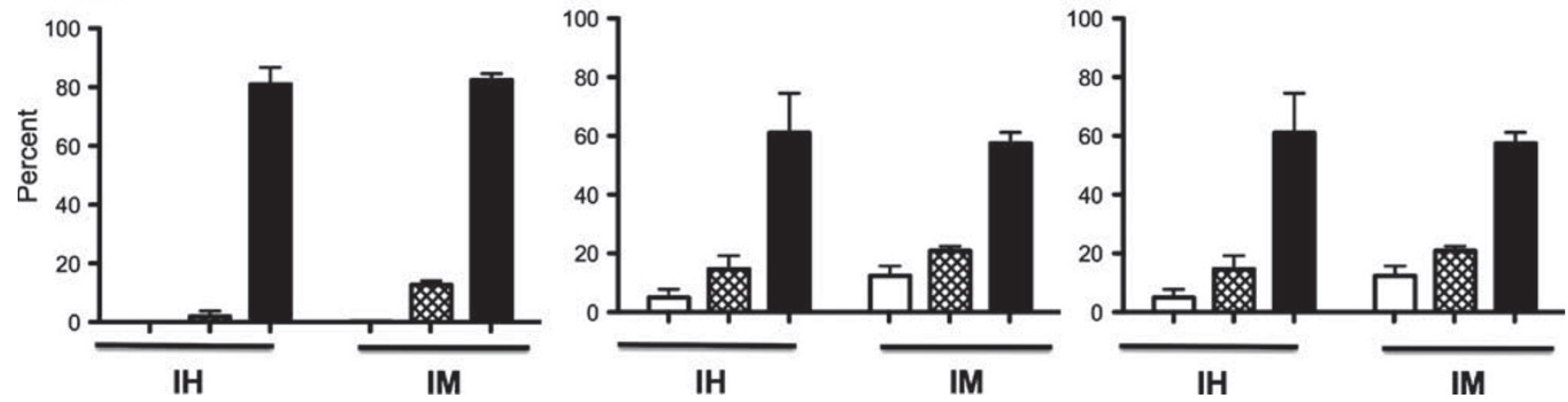

Figure 3. Effector and memory differentiation of activated CD8 T cells. Expansion and contraction of short-lived effector cells (SLECs) and memory precursor effector cells (MPECs) in (a) PBMC and (b) other lymphoid and non-lymphoid tissues. (c) Distribution of effector and memory CD8 T cells from intrahepatically (I.H.)- and intramuscularly (I.M.) immunized mice at days 21 and 84 post infection. Data are representative of three independent experiments with four mice per group. 


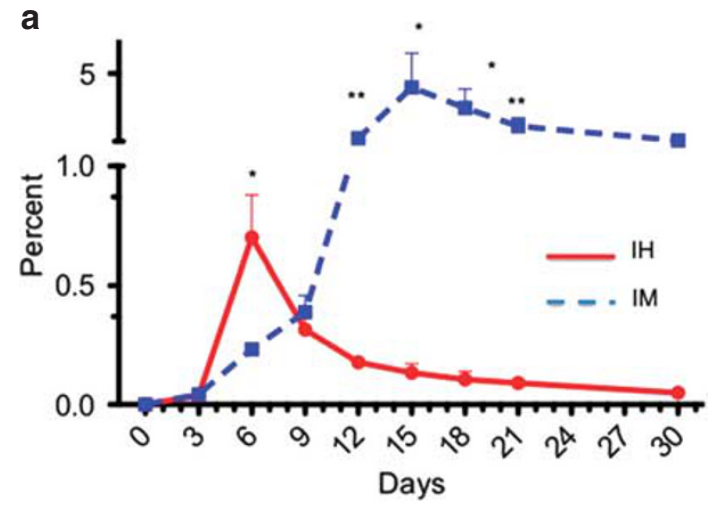

b

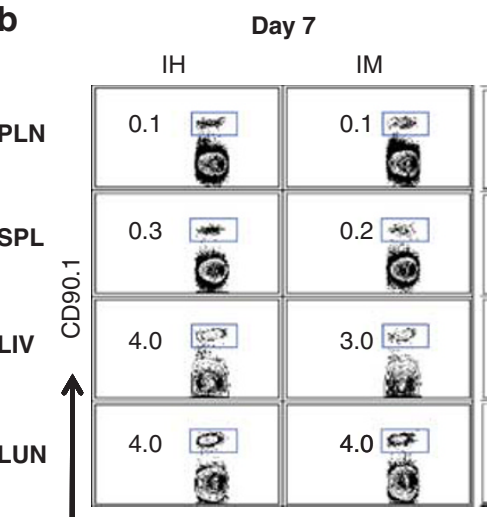

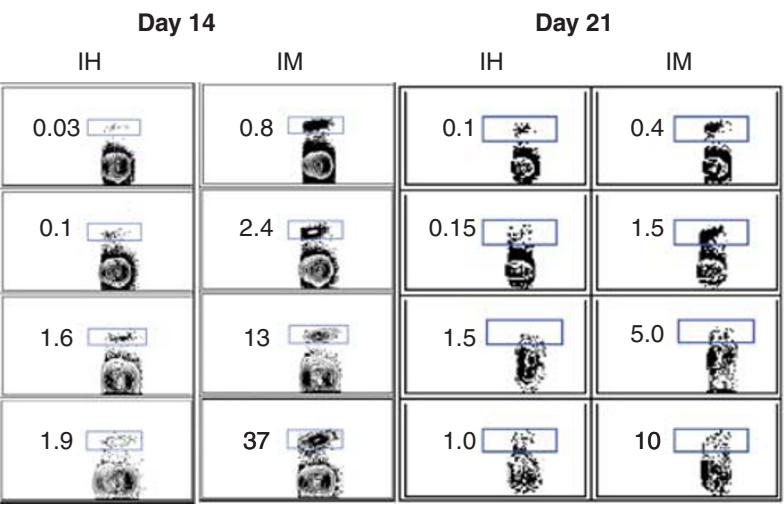
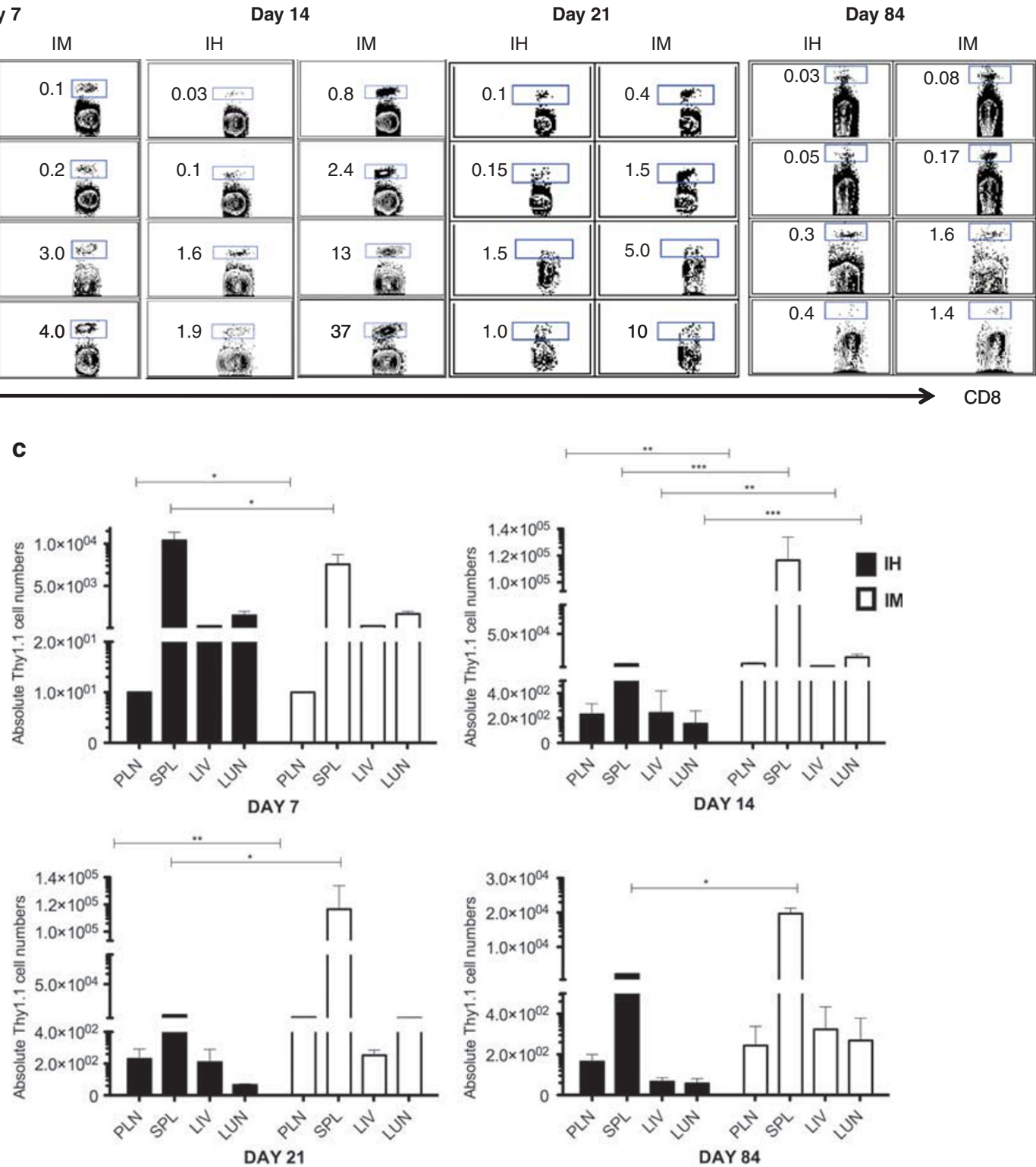

Figure 4. Effector CD8 T cells frequency magnitude and distribution. Frequency kinetics of the GP33-specific CD8 T-cell response in (a) PBMC and (b, c) lymph nodes, spleen (SPL), liver (LIV) and lung (LUN) following immunization. Data are representative of three independent experiments with four mice per group.

CD8 T cells that will commit to become memory T cells. ${ }^{24}$ Cells within a polyclonal response can be grouped into SLECs and MPECs based on their expression levels of the killer cell marker;
KLRG1 among other surface markers. We investigated the difference in I.H. vs I.M.-primed CD8 T cells' capacity to differentiate into SLEC and MPEC based on immunization route. 
The CD8 T cells in the blood of the I.H. group exhibited more SLEC subsets at earlier time points, whereas the MPEC subset increased only 12 days post immunization, when the SLEC begin to die off. A similar trend was seen in lymphoid and other non-lymphoid tissues. This early increase in KLRG ${ }^{\text {hi }}$ cells in the I.H.-immunized group was in contrast to what was observed in the I.M.-immunized animals (Figures $3 a$ and $b$ ).

Although these markers allowed us to identify the CD8 T-cell population that has the potential to become memory cells, it was not clear if they actually became memory CD8 T cells without additional study. Using CD44, IL-7R $\alpha$ and L-selectin, we further categorized the Thy1.1 cells into effector, effector-memory and central memory CD8 T cells (Figure 3c). Flow cytometric analysis of CD8 T cells from the lymph nodes, SPL and LIV on days 21 and 84, indicated a similar distribution of effector, effector-memory and central memory cells between the I.H. and I.M. groups (Figure 3c).

Activated CD8 T cells in I.H.-immunized mice experienced a swift expansion and contraction phase

Although CD8 T cells from both immunized groups differentiated into effector cells and memory cells, it was unclear whether they undergo this transition with the same frequency distribution. We explored the frequency of CD8 T-cell responses induced by I.H. in contrast to those induced by I.M. Following immunization, T-cell expansion from I.H. rapidly peaked on day 6 with $0.7 \%$ of Ag-specific cells detected in the blood. This early expansion was followed by a rapid contraction phase. At day 9 post infection, $57 \%$ of the Ag-specific cells were already dead and the percent contraction increased to $82 \%$ by week 2 . In the I.M. group, only $0.2 \%$ Ag-specific cells were detected on day 6 . However, an increase in Ag-specific CD8 T cells (to $\sim 4.2 \%$ ) was observed at day 15 post infection. Furthermore, the Ag-specific CD8 T cells in I.M. group underwent a gradual contraction over the next 3 weeks (Figure 4a).

To evaluate the tissue distribution of Ag-specific cells in lymphoid and non-lymphoid tissues, we calculated the absolute numbers of Thy1.1 cells in these tissues (Figures $4 a$ and c). We observed similar expansion-contraction phases between groups in these tissues as seen in the blood (Figure 4a). The rapid and robust contraction phase in the I.H. group after expansion led to a low frequency of effector CD8 T cells, which persisted several weeks after immunization. The I.M. group on the other hand, exhibited $\sim 1.6 \%$ of the activated cells still in the LIV, 12 weeks after immunization.

The decrease in CTL frequency in I.H.-immunized mice leds to defective viral clearance.

In order to examine the effects of these two different immune phenotypes in protection, we challenged the animals 3 weeks post immunization. At this time point $0.15 \%$ Ag-specific CD8 T cells can be detected in the SPL of the I.H.-immunized group (compared with $1.5 \%$ of the I.M. group). When killing assay was performed, $<2 \%$ of the relevant $\mathrm{Ag}$-pulsed target cells were eliminated in the I.H. group. This is in sharp contrast to the I.M. group, which showed killing of about $75 \%$ of the target cells (Figure 5a). We further allowed the transferred target cells to reside in the recipient mice for up to $72 \mathrm{~h}$, but we did not observe any improvement in killing (Figure 5a). This lack of cytolytic activity was also observed in the lymph nodes, SPL, LIV and LUN, illustrating that the vaccine-induced effector CD8 T cells were not selectively sequestered in any particular tissue (Figure $5 \mathrm{~b}$ ).

To confirm that the lack of target cell elimination in the I.H. group is not due to defective LIV-primed memory CD8 T cells, we transferred $3.5 \times 10^{4} \mathrm{Ag}$-specific cells from each group into naive mice and evaluated their cytolytic activity in the recipient mice. Interestingly, none of the recipient groups were able to eliminate the peptide-pulsed target cells (Figure 5c).
Four to six weeks post immunization, both groups were challenged with 200 plaque-forming units of LCMV Armstrong by the I.C. route to test functional effector function toward a pathogen challenge. Figure $5 d$ displays the data from the challenge study and survival for each group of mice. Although the I.M. immunization resulted in protection of $80 \%$ of challenged animals surviving, all naive mice and I.H.-vaccinated animals succumbed to infection.

Naive CD8 T cells in I.H.-immunized group encounter cognate Ags outside the lymph nodes

We next investigated the mechanism of induction of CD8 T cells during intraheptatic immunization. As started previously, there is an induction of the naïve P14 CD8 T cells through I.H. immunization. However, it was not clear where these CD8 T cells were being activated. To examine if the Ag was localized or transported and presented in regional lymphoid tissues, a group of naive P14-recipient mice were treated with L-selectin (CD62L) antibody before immunization. This treatment prevents trafficking of naive T cells from the lymph nodes ${ }^{14}$ and depletion of naive T cells from lymph nodes. Consistent with previous data, ${ }^{25}$ anti-L-selectin treatment led to severe naive T-cell depletion in the lymph nodes. Anti-L-selectin treatment did not change the priming of transferred P14 T-cell receptor transgenic lymphatic CD8 T cells after I.H. immunization with cognate Ag. The priming was supported by observed Ag-specific proliferation and CD8 T-cell expansion (Figure 6), showing that activation was occurring outside the lymph nodes of the mice. Acknowledging that the activation could be occurring in the SPL (as entry of some naive lymphocytes into the SPL is L-selectin independent), we treated the mice with an immunomodulating compound, (FTY720), which sequesters naive lymphocytes in the lymph nodes, thus preventing interaction of these cells with the I.H.-transfected hepatocytes. Sequestering of naive lymphocytes in the lymph nodes prevented the priming of transferred P14 T-cell receptor transgenic CD8 T-cell induction and proliferation (Figure 6). Together, these data suggest that LIV transfection through $\mathrm{HI}$ encourages extralymphatic priming of the transgenic CD8 T cells. These studies support that the DNA-transfected cognate Ag-expressing hepatocytes successfully induced activation of naive P14 CD8 T cells, (Figure 6) as has been previously suggested from transplant, adenovirus and AAV models. ${ }^{26-28}$

\section{DISCUSSION}

The recent resurgence in popularity of synthetic DNA vaccination has been attributed to their ability to elicit strong humoral and cellular responses without the safety, stability and expense concerns associated with live attenuated and recombinant protein vaccines. It is therefore important to study this platform in the development of immune therapies for chronic infections such as HBV and HCV. These two hepatotropic pathogens are known to be the leading cause of one of the deadliest forms of cancer, HCC.

Most of the experimental DNA vaccines have been delivered I.M. and they do not elicit significant immune responses in mucosal and/or other tissues that are not associated with the peripheral lymphoid tissues. It may be important to utilize the microenvironment of regional lymph nodes to present effector cells with homing instructions by using a delivery system that will make these regional lymph nodes the site of activation for immune cells. In this study, we report an unexpected defect in the initial induction of adaptive immunity following I.H. immunization of P14 chemaric mice. We believe that the unconventional priming of naive CD8 T cells in this type of immunization compromises the cells' expansion capacity, resulting in a 3-500fold decrease of effector cell frequency and 2-9-fold decrease of memory CD8 T-cell frequency in multiple tissues. Although T cells 
b

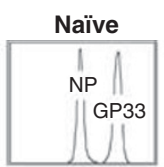

a

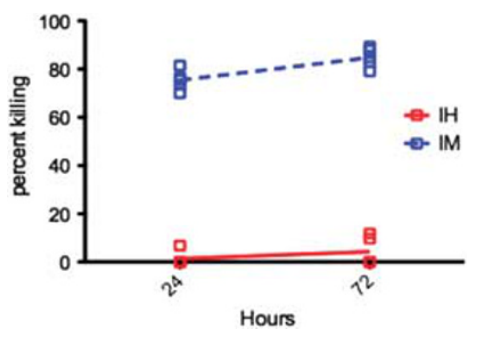

C
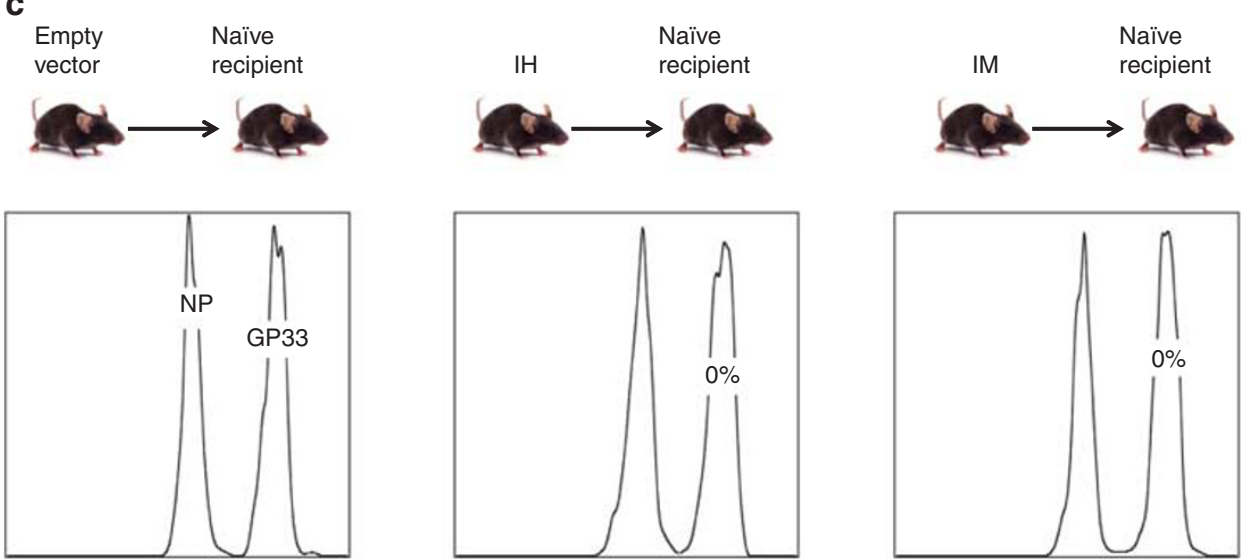

d

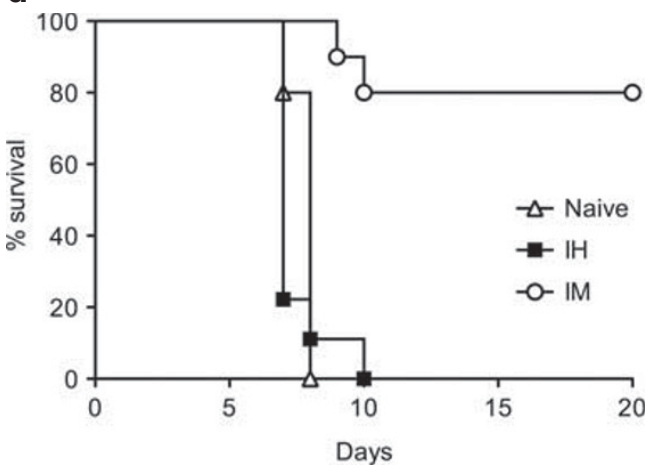

Figure 5. Lack of CTL-mediated cytotoxicity in intrahepatically (I.H.) immunized mice at late time points. The ability of I.H.-immunized mice to specifically remove antigen (Ag)-pulsed target cells in vivo at week 1 was diminished before day 21. Percent in vivo killing in (a) PBMC and (b) the spleen (SPL), lymph nodes, liver (LIV) and lung (LUN) for I.H. and intramuscular (I.M.) mice at week 3 post immunization. (c) Cytolytic activity in the SPL of naive mice that have respectively received equal frequencies $(30000)$ of activated $\mathrm{D}^{\mathrm{b}} \mathrm{GP} 33^{+}$-specific CD8 $\mathrm{T}$-cell from I.H.- and I.M.-immunized mice. Data are representative of three independent experiments with four mice per group. (d) Thirty days after immunization, groups of naive, hydrodynamically injected and I.M. immunized mice were challenged with lethal dose of LCMV by I.C. infection ( $n=10-15$ mice per group). Following I.C. infection, mice were monitored for mortality. Data are representative of three independent experiments with four mice per group.

from this immunization group resulted in early effector differentiation, the overall quality of the effector functions, polyfunctionality and cytotoxicity, were somewhat comparable to CD8 $\mathrm{T}$ cells primed in lymphoid tissues. The memory cells' poor Ag clearance and poor viral control illustrate a low-CTL frequency.
Primary CD8 T-cell activation by liver cells have been described by several independent groups using in vivo and in vitro models. ${ }^{26,29,30}$ The fate of CD8 T cells primed in the liver has been a subject of much investigation in an effort to explain the failure of LIV protective immunity in chronic liver infections, such 


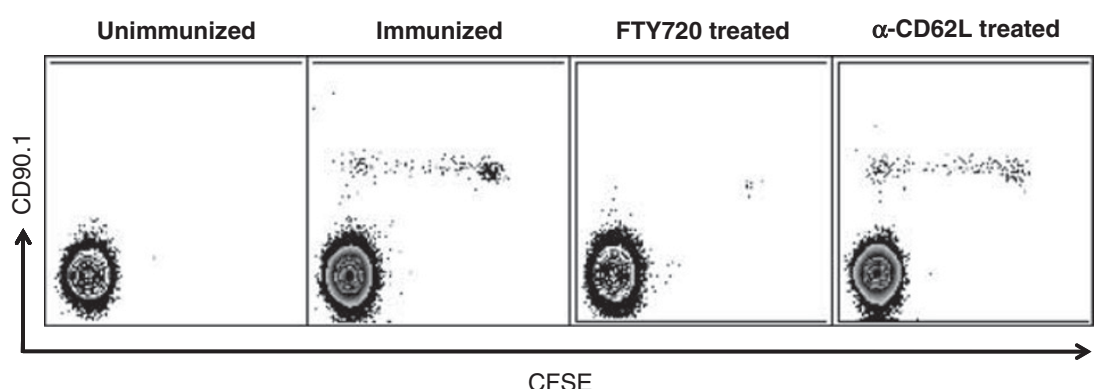

CFSE

Figure 6. Expression of transgene product in the liver causes extra-lymphatic CD8 T cells priming. Lymph node naive CD8 $T$ cells priming indicated by $\mathrm{D}^{\mathrm{b}} \mathrm{GP} 33^{+}$-specific/Thy1.1 CD8 T-cell division and expansion. Treatment with FTY720, an immunosuppressive drug that sequesters circulating lymphocytes in lymph nodes, abrogated the cell activation. However, preventing lymph node entry of naive lymphocytes using anti-CD62L had no effect on $\mathrm{D}^{\mathrm{b}} \mathrm{GP} 33^{+}$-specific CD8 T-cell induction. These results confirm extra-lymphatic activation of CD8 T cells following HI of naked DNA. Data are representative of three independent experiments with three mice per group.

as HBV, HCV and malaria. Using liver transplantation and transgenic Ag models, Bertolino et al., ${ }^{31-33}$ suggested that liverprimed CD8 T cells underwent early Bim-dependent apoptotic death. Other groups studying this question with viral vectors, such as adenovirus ${ }^{27}$ and $A A V^{34}$ reported full differentiation with some defective antiviral activities. The outcomes of these studies are important in interpreting and understanding the present data. To gain insight into how Ag expression in the liver can help migrate effector T cells preferentially into the liver surroundings, and to compare the differentiation and memory programming of these $T$ cells to normal I.M. immunization, a unique model that establishes $\mathrm{Ag}$ infection but eliminates chronic Ag expression was needed. Here, we used a hydrodynamic DNA plasmid injection approach to establish an acute liver 'infection' model that allowed us to address this question. Hydrodynamic-based transfection normally results in the rapid uptake of DNA molecules into the cytosol of hepatocytes ${ }^{35}$ and detectable $\mathrm{Ag}$ expression in the liver within a few hours. The Ag expression kinetics are comparable to what is observed in skeletal muscle after conventional I.M. injection. ${ }^{36}$ We observed that such expression was restricted to the liver and lasted for $\sim 4$ days, mimicking acute infection. The level of transgene expression is likely to represent the expression of viral $\mathrm{Ag}$ in the liver during hepatotropic pathogenic infection. Furthermore, there was no significant elevation in blood alanine aminotransferase, an enzyme used to measure LIV damage.

Using this model, LCMV D ${ }^{\mathrm{b}}$ GP33-specific CD8 T-cell priming in P14 chimeric mice was confirmed by analysis of the upregulation of effector molecules. However, to clear pathogenic infection, CD8 T-cell priming alone is not sufficient. The activated CD8 T cell should be able to survive long enough to differentiate into a mature effector cell that possesses antiviral properties. After observing priming of CD8 T cells in both immunization groups, we observed rapid upregulation of pro-apoptotic markers (Supplementary Figure 1) in the I.H. group, and therefore believed that the CD8 T cells from the I.H. group may lack the ability to survive long enough to have a significant impact on viral resolution. This idea is based on the knowledge that Bim-dependent deletion of CD8 T cells is a link to the inability of HBV-specific CD8 T cells to control viral persistence in chronic infections. ${ }^{37}$ In the present study, we observed an early proliferative response of naive CD8 T cells in the I.H. group when compared with the traditional I.M. immunization model, where T-cell activation is occurring in the draining lymph nodes. We noticed that the early cell division of Agspecific CD8 T cells in the I.H. group was consistent with prior studies, which shows the kinetics of initial I.H. priming to be more rapid than by secondary lymphoid tissue-dependent priming. ${ }^{27}$ The early proliferation of $D^{b}$ GP33-specific CD8 T cells in the I.H. group was followed by significant untimely effector cell contraction. In this case, the CD8 T cells expanded early, but the cell frequency at their peak of expansion was several folds lower than CD8 T cells primed in the draining lymph nodes. As expected, the pro-apoptotic marker, Bim, was upregulated in the I.H. group during this contraction phase. The Bim levels in the I.M. group eventually caught up with the I.H. group during its peak of expansion (Supplementary Figure 1A). Interestingly, the survival signal, Bcl-2, in the I.H. group was downregulated in the first week when Bim was high, but increased during the second and third weeks post immunization (Supplementary Figure 1B). Although this data support that CD8 T cells from I.H. immunization, albeit hastily, experience the same death and survival phases as CD8 T cells from I.M. immunization, it also provide an inkling of induction of a different CD8 T-cell activation pattern.

In addition to the phenotypic examination I.H. and I.M. CD8 $T$ cells' effector differentiation status, we also analyzed their functional properties. Although some prior studies reported full functional differentiation of CD8 T cells when Ag is expressed in the liver, ${ }^{34}$ a more comparative analysis of these effector cells disclosed defective antiviral activity. ${ }^{27}$ Our findings show that the effector CD8 T cells from the I.H. group can secrete antiviral cytokines. Though they were inferior single producers, their polyfunctionality, a property reported to correlate with protection in specific models, ${ }^{38}$ was very similar to the control group in all tissues except the lymph nodes. It is noteworthy that for both immunized groups effector CD8 T cells recovered from the LIV were less functional compared with those found in the same animals' spleen and lymph nodes. Therefore, prior work and the data presented here support that the liver environment has a crucial role in effector T-cell suppression. At earlier time points, the I.H. group mounted a strong cell-mediated cytotoxic effect upon Ag re-encounter. This data support that effector CD8 T cells recovered from I.H. immunization early can be as effective as those from traditional I.M. immunization.

Our results also reveal that a subset of effector cells (MPEC), which are likely to become long-lived memory cells, can be detected in the I.H.-immunized group's effector CD8 T-cell population as early as 1 week post immunization. Although CD8 T cells from I.H. differentiate into memory cells quicker than I.M.-immunized group, the distribution of effector, effectormemory and central memory cells were similar in all tissues within the groups.

We evaluated recall and effector differentiation of these longlived memory CD8 T cells in both lymphoid and non-lymphoid tissues. The I.M. group responded robustly and eliminated target cells from all tissues upon secondary challenge. In contrast, the I.H. group was deficient in killing these Ag-pulsed target cells. This lack of killing was likely associated with the low frequency of effector-memory cells in the I.H. group. Although the I.H. group's 
memory precursors comprise about $16 \%$ of peak effector cells in the I.H. group, the frequency was still very low because of its lower effector frequency. In support, hydrodynamic-immunized mice repeatedly succumbed to lethal dose LCMV I.C. challenge.

To investigate the difference we observed in the expansioncontraction phase and the decrease in frequency in the I.H. group, we examined differences in the initial induction of CD8 T cells in both groups. Using models that ensure the exclusion of CD8 T-cell priming in lymphoid tissues, we observed proliferation and expansion of $\mathrm{GP}_{3}{ }^{+} /$Thy 1.1 cells in P14 chimeras after I.H. immunizing mice with cognate viral Ag. Collectively, these data show that hydrodynamically injecting DNA plasmid into mice causes lymphoid-independent CD8 T-cell activation.

In summary, these results provide evidence that the lack of recall response needed to protect mice from secondary challenge after I.H. immunization is directly associated with a defect in the initial induction of the Ag-driven response. This correlation can be made to human studies in which the lack of viral clearance in chronic HBV and HCV infections have been linked with the low number of HBV or HCV-specific CD8 T cells detected in the blood. ${ }^{39,40}$ Although further studies are needed, the data support that it is important to concentrate on improving traditional I.M. immunization that will preferentially traffic effective cellular immune cells into the liver for immunotherapies to target hepatotropic pathogens.

\section{ABBREVIATIONS}

HBV, hepatitis B; HCV, hepatitis $\mathrm{C}$; $\mathrm{HI}$, hydrodynamic injection; I.C., intracranial; I.H., intrahepatic; I.M., intramuscular; LIV, liver; MPEC, memory precursor effector cells; SLEC, short-lived effector cells

\section{CONFLICT OF INTEREST}

DBW has grant funding, participates in industry collaborations, has received speaking honoraria and fees for consulting. This service includes serving on scientific review committees and advisory boards. Remuneration includes direct payments or stock or stock options and in the interest of disclosure; therefore, he notes potential conflicts associated with this work with Pfizer, Bristol Myers Squibb, Inovio, Merck, VGXI, Aldevron and possibly others. Licensing of technology from his laboratory has created over 100 jobs in the private sector in the biotech/pharma industry. The other authors declare no competing financial interests.

\section{ACKNOWLEDGEMENTS}

We are grateful to Dr John Wherry for providing the transgenic mice and Dr Rafi Ahmed for the DNA plasmid.

\section{REFERENCES}

1 Ahmed R, Gray D. Immunological memory and protective immunity: understanding their relation. Science 1996; 272: 54-60.

2 Kalia V, Sarkar S, Gourley TS, Rouse BT, Ahmed R. Differentiation of memory B and T cells. Curr Opin Immunol 2006; 18: 255-264.

3 Germain RN, Robey EA, Cahalan MDA. Decade of imaging cellular motility and interaction dynamics in the immune system. Science 2012; 336: 1676-1681.

4 Stremmel C, Sienel W, Eggeling S, Passlick B, Slavin A. Inhibition of T-cell homing by down-regulation of $\mathrm{CD} 62 \mathrm{~L}$ and the induction of a Th-2 response as a method to prevent acute allograft rejection in mice. Eur J Cardiothorac Surg 2006; 30: 362-369.

5 Beltman JB, Marée AF, Lynch JN, Miller MJ, De Boer RJ. Lymph node topology dictates T-cell migration behavior. J Exp Med 2007; 204: 771-780.

6 Figge MT, Garin A, Gunzer M, Kosco-Vilbois M, Toellner K-M, Meyer-Hermann M. Deriving a germinal center lymphocyte migration model from two-photon data. J Exp Med 2008; 205: 3019-3029.

7 Linderman JJ, Riggs T, Pande M, Miller M, Marino S, Kirschner DE. Characterizing the dynamics of CD4 + T-cell priming within a lymph node. J Immunol 2010; 184 2873-2885

8 Butcher EC, Williams M, Youngman K, Rott L, Briskin M. Lymphocyte trafficking and regional immunity. Adv Immunol 1999; 72: 209-253.

9 Masopust D, Vezys V, Marzo AL, Lefrançois L. Preferential localization of effector memory cells in nonlymphoid tissue. Science 2001; 291: 2413-2417.
10 Weninger W, Manjunath N, Von Andrian UH. Migration and differentiation of CD8 + T cells. Immunol Rev 2002; 186: 221-233.

11 Mora JR, Bono MR, Manjunath N, Weninger W, Cavanagh LL, Rosemblatt M et al. Selective imprinting of gut-homing T cells by Peyer's patch dendritic cells. Nature 2003; 424: 88-93.

12 Kantele A, Zivny J, Häkkinen M, Elson CO, Mestecky J. Differential homing commitments of antigen-specific $\mathrm{T}$ cells after oral or parenteral immunization in humans. J Immunol 1999; 162: 5173-5177.

13 Masopust D, Choo D, Vezys V, Wherry EJ, Duraiswamy J, Akondy R et al. Dynamic T-cell migration program provides resident memory within intestinal epithelium. J Exp Med 2010; 207: 553-564.

14 Harp JR, Gilchrist MA, Onami TM, Memory T. Cells are enriched in lymph nodes of selectin-ligand'Äideficient mice. J Immunol 2010; 185: 5751-5761.

15 Zhang G, Budker V, Wolff JA. High levels of foreign gene expression in hepatocytes after tail vein injections of naked plasmid DNA. Human Gene Ther 1999; 10: 1735-1737.

16 Yan J, Harris K, Khan AS, Draghia-Akli R, Sewell D, Weiner DB. Cellular immunity induced by a novel HPV18 DNA vaccine encoding an E6/E7 fusion consensus protein in mice and Rhesus macaques. Vaccine 2008; 26: 5210-5215.

17 Masopust D, Ha S-J, Vezys V, Ahmed R. Stimulation history dictates memory CD8 T cell phenotype: implications for prime-boost vaccination. J Immunol 2006; 177 831-839.

18 Murali-Krishna K, Altman JD, Suresh M, Sourdive DJD, Zajac AJ, Miller JD et al. Counting antigen-specific CD8 T Cells: a reevaluation of bystander activation during viral infection. Immunity 1998; 8: 177-187.

19 Barber DL, Wherry EJ, Ahmed R. Cutting edge: rapid in vivo killing by memory CD8 T cells. J Immunol 2003; 171: 27-31.

20 Durward MA, Harms J, Magnani DM, Eskra L, Splitter GA. Discordant Brucella melitensis antigens yield cognate CD8 + T cells in vivo. Infect Immun 2010; 78: 168-176.

21 Shedlock DJ, Talbott KT, Cress C, Ferraro B, Tuyishme S, Mallilankaraman K et al. A highly optimized DNA vaccine confers complete protective immunity against high-dose lethal lymphocytic choriomeningitis virus challenge. Vaccine 2011; 29: 6755-6762.

22 Yang PL, Althage A, Chung J, Chisari FV. Hydrodynamic injection of viral DNA: A mouse model of acute hepatitis B virus infection. Proc Natl Acad Sci USA 2002; 99 13825-13830.

23 Osborn MJ, McElmurry RT, Lees CJ, DeFeo AP, Chen ZY, Kay MA et al. Minicircle DNA-based gene therapy coupled with immune modulation permits long-term expression of [alpha]-l-iduronidase in mice with mucopolysaccharidosis type I. Mol Ther 2011; 19: 3

24 Amoah S, Yammani RD, Grayson JM, Alexander-Miller MA. Changes in functional but not structural avidity during differentiation of CD8 + effector cells in vivo after virus infection. J Immunol 2012; 189: 638-645.

25 Lepault F, Gagnerault M-C, Faveeuw C, Boitard C. Recirculation, phenotype and functions of lymphocytes in mice treated with monoclonal antibody MEL-14. Eur $J$ Immunol 1994; 24: 3106-3112.

26 Bertolino $P$, Trescol-Biémont $M-C$, Rabourdin-Combe C. Hepatocytes induce functional activation of naive $\mathrm{CD} 8+\mathrm{T}$ lymphocytes but fail to promote survival. Eur J Immunol 1998; 28: 221-236.

27 Lukens JR, Dolina JS, Kim TS, Tacke RS, Hahn YS. Liver is able to activate naive CD8 + T cells with dysfunctional anti-viral activity in the murine system. PLOS ONE 2009; 4: e7619.

28 Wuensch SA, Spahn J, Crispe IN. Direct, help-independent priming of CD8 + T cells by adeno-associated virus-transduced hepatocytes. Hepatology 2010; 52: 1068-1077.

29 Bertolino P, Bowen DG, McCaughan GW, Fazekas de St. Groth B. Antigen-specific primary activation of CD8 + T cells within the liver. J Immunol 2001; 166: $5430-5438$.

30 Crispe IN. Liver antigen-presenting cells. J Hepatol 2011; 54: 357-365.

31 Bertolino P, Trescol-Biémont MC, Thomas J, Fazekas De St Groth B, Pihlgren M, Marvel J et al. TDeath by neglect as a deletional mechanism of peripheral tolerance. Int Immunol 1999; 11: 1225-1238.

32 Holz LE, Benseler V, Bowen DG, Bouillet P, Strasser A, O'Reilly L et al. Intrahepatic murine CD8 T-cell activation associates with a distinct phenotype leading to bimdependent death. Gastroenterology 2008; 135: 989-997.

33 Holz LE, Benseler V, Vo M, McGuffog C, Van Rooijen N, McCaughan GW et al. Naive CD8 T-cell activation by liver bone marrow-derived cells leads to a 'Äúneglected'Äù IL-2low Bimhigh phenotype, poor CTL function and cell death. J Hepatol 57: 830-836.

34 Wuensch SA, Pierce RH, Crispe IN. Local intrahepatic CD8 + T-cell activation by a non-self- antigen results in full functional differentiation. $J$ Immunol 2006; 177: 1689-1697.

35 Andrianaivo $F$, Lecocq $M$, Wattiaux-De Coninck $S$, Wattiaux $R$, Jadot $M$ Hydrodynamics-based transfection of the liver: entrance into hepatocytes of DNA 
that causes expression takes place very early after injection. J Gene Med 2004; 6: 877-883.

36 Wolff J, Dowty ME, Jiao S, Williams P. Expression of naked plasmids by cultured myotubes and entry of plasmids into $T$ tubules and caveolae of mammalian skeletal muscle. Cell Science 1992; 103: 1249-1259.

37 Lopes AR, Kellam P, Das A, Dunn C, Kwan A, Turner J et al. Bim-mediated deletion of antigen-specific CD8 + T cells in patients unable to control HBV infection. J Clin Invest 2008; 118: 1835-1845.

38 Betts MR, Nason MC, West SM, De Rosa SC, Migueles SA, Abraham J et al. HIV nonprogressors preferentially maintain highly functional HIV-specific CD8 + T cells. Blood 2006; 107: 4781-4789.
39 Sobao Y, Tomiyama H, Sugi K, Tokunaga M, Ueno T, Saito S et al. The role of hepatitis $B$ virus-specific memory CD8 T cells in the control of viral replication. J Hepatol 2002; 36: 105-115.

40 Sobao Y, Tomiyama H, Nakamura S, Sekihara H, Tanaka K, Takiguchi M. Visual demonstration of hepatitis $C$ virus-specific memory CD8 + T-cell expansion in patients with acute hepatitis C. Hepatology 2001; 33: 287-294.

(c) $\Theta$ This work is licensed under a Creative Commons AttributionCon ${ }_{\mathrm{BY}}$ ND Nommercial-NoDerivs 3.0 Unported License. To view a copy of this license, visit http://creativecommons.org/licenses/by-nc-nd/3.0/

Supplementary Information accompanies the paper on Cancer Gene Therapy website (http://www.nature.com/cgt) 